\title{
The Poisson structure of the mean-field equations in the $\Phi^{4}$ theory
}

\author{
Cécile Martin \\ Groupe de Physique Théorique de l'Institut de Physique Nucléaire \\ Institut de Physique Nucléaire, \\ F-91406, Orsay Cedex, France*
}

December 13, 2017

\begin{abstract}
We show that the mean-field time dependent equations in the $\Phi^{4}$ theory can be put into a classical non-canonical hamiltonian framework with a Poisson structure which is a generalization of the standard Poisson bracket. The Heisenberg invariant appears as a structural invariant of the Poisson tensor.
\end{abstract}

${ }^{*}$ E-mail address : martinc@ipno.in2p3.fr 
Poisson Structure in $\Phi^{4}$ Theory 


\section{Introduction}

The purpose of this paper is to give a formulation of the mean-field dynamical equations in a bosonic quantum field theory in terms of generalized Poisson brackets. The time-dependent Hartree-Bogoliubov (TDHB) equations are dynamical equations for the expectations values of one and two field operators. They have been used to study nonequilibrium evolution in $\Phi^{4}$ theory [1]. They are quantum dynamical equations. However, we will show that they can be put into a classical non-canonical hamiltonian framework with a Poisson structure which is a generalization of the standard Poisson bracket.

Non-canonical Poisson brackets have been found to be usefull in different contexts, including plasma and fluid dynamics [2] and condensed matter physics [3]. In relativistic field theories, it has been shown how to write the field equations in covariant Poisson bracket form [4]. In non-relativistic quantum theory, a Poisson structure has been recognized for the time-dependent mean-field dynamics of a system of fermions [5] or a system of bosons [6]. A Poisson structure is especially useful to find structural invariants and to study stability properties. We will show how this approach can be developped in quantum field theory.

We consider the $\Phi^{4}$ theory. In section 2, we introduce the dynamical variables of interest and we recall the TDHB equations which can be written in a compact form [7]. We define the Poisson tensor in section 3 and we show the Poisson structure of the TDHB equations. Another definition of the Poisson tensor from a Lie algebra is considered in section 4. In section 5, we recognize the Heisenberg invariant as a structural invariant of the Poisson tensor. Finally, in section 6, we write the linearized dynamical equations arround the HB static solution.

\section{The mean-field equations in the $\Phi^{4}$ theory :}

We consider a self-interacting scalar field in a Minkowski metric described by the Hamiltonian $H=\int d^{d} x \mathcal{H}(\vec{x})$ with

$$
\mathcal{H}(\vec{x})=\frac{1}{2} \Pi^{2}(\vec{x})+\frac{1}{2}(\vec{\nabla} \Phi(\vec{x}))^{2}+\frac{m_{0}^{2}}{2} \Phi^{2}(\vec{x})+\frac{b}{4} \Phi^{4}(\vec{x}) .
$$

We work in $d$ spatial dimensions. The constants $m_{0}$ and $b$ are respectively the bare mass and the bare coupling constant. $\Phi(\vec{x})$ and $\Pi(\vec{x})$ are the field operators in the Schrödinger representation. The initial state of the system is supposed to be given and equal to a statistical operator $D\left(t_{0}\right)$ which may represent a thermal equilibrium or a non-equilibrium situation. At zero temperature, it reduces to a projection operator. 
A time-dependent variational principle [8] and the choice of gaussian operators for the trial space allow to derive time-dependent mean-field equations for the expectation values [1, 7, 8]. A gaussian density-like operator $\mathcal{D}(t)$ is completly characterized by a vector $\alpha(\vec{x}, t)$ and a matrix $\Xi(\vec{x}, \vec{y}, t)$ defined by :

$$
\begin{gathered}
\alpha(\vec{x}, t)=\left(\begin{array}{c}
\varphi(\vec{x}, t) \\
-i \pi(\vec{x}, t)
\end{array}\right), \\
\Xi(\vec{x}, \vec{y}, t)=\left(\begin{array}{cc}
2 G(\vec{x}, \vec{y}, t) & -i T(\vec{x}, \vec{y}, t) \\
-i T(\vec{y}, \vec{x}, t) & -2 S(\vec{x}, \vec{y}, t)
\end{array}\right),
\end{gathered}
$$

where

$$
\begin{gathered}
\varphi(\vec{x}, t)=\frac{1}{n(t)} \operatorname{Tr}(\mathcal{D}(t) \Phi(\vec{x})) \\
\pi(\vec{x}, t)=\frac{1}{n(t)} \operatorname{Tr}(\mathcal{D}(t) \Pi(\vec{x})), \\
G(\vec{x}, \vec{y}, t)=\frac{1}{n(t)} \operatorname{Tr}(\mathcal{D}(t) \bar{\Phi}(\vec{x}) \bar{\Phi}(\vec{y})), \\
S(\vec{x}, \vec{y}, t)=\frac{1}{n(t)} \operatorname{Tr}(\mathcal{D}(t) \bar{\Pi}(\vec{x}) \bar{\Pi}(\vec{y})), \\
T(\vec{x}, \vec{y}, t)=\frac{1}{n(t)} \operatorname{Tr}(\mathcal{D}(t)(\bar{\Phi}(\vec{x}) \bar{\Pi}(\vec{y})+\bar{\Pi}(\vec{y}) \bar{\Phi}(\vec{x}))), \\
n(t)=\operatorname{Tr} \mathcal{D}(t),
\end{gathered}
$$

and, for any operator $O: \bar{O}=O-\operatorname{Tr}(\mathcal{D}(t) O)$.

The time-dependent Hartree-Bogoliubov (TDHB) equations for the $\Phi^{4}$ theory can be written in the following compact form [7] :

$$
\begin{gathered}
i \dot{\alpha}=\tau w, \\
i \dot{\Xi}=-[(\Xi+\tau) \mathcal{H}(\Xi-\tau)-(\Xi-\tau) \mathcal{H}(\Xi+\tau)] .
\end{gathered}
$$

where $\tau$ is the $2 \times 2$ matrix

$$
\tau=\left(\begin{array}{cc}
0 & 1 \\
-1 & 0
\end{array}\right)
$$

The vector $w$ and the matrix $\mathcal{H}$ are defined from the variation of

$$
\begin{gathered}
<H>=\frac{1}{n(t)} \operatorname{Tr}(\mathcal{D}(t) H)=\int d^{d} x \mathcal{E}(\vec{x}, t) \\
\delta<H>=\int d^{d} x \tilde{w}(\vec{x}, t) \delta \alpha(\vec{x}, t)-\frac{1}{2} \int d^{d} x d^{d} y \operatorname{tr}(\mathcal{H}(\vec{x}, \vec{y}, t) \delta \Xi(\vec{y}, \vec{x}, t)) .
\end{gathered}
$$

$\mathcal{E}(\vec{x}, t)$ is the mean-field energy density. For the Hamiltonian (2.1), we have :

$$
w_{1}(\vec{x}, t)=\left(-\Delta+m_{0}^{2}+\frac{b}{6} \varphi^{2}(\vec{x}, t)+\frac{b}{2} G(\vec{x}, \vec{x}, t)\right) \varphi(\vec{x}, t)
$$




$$
\begin{gathered}
w_{2}(\vec{x}, t)=i \pi(\vec{x}, t) \\
\mathcal{H}_{11}(\vec{x}, \vec{y}, t)=-\frac{1}{2}\left(-\Delta+m_{0}^{2}+\frac{b}{2} \varphi^{2}(\vec{x}, t)+\frac{b}{2} G(\vec{x}, \vec{x}, t)\right) \delta(\vec{x}-\vec{y}) \\
\mathcal{H}_{22}(\vec{x}, \vec{y}, t)=\frac{1}{2} \delta(\vec{x}-\vec{y}) \\
\mathcal{H}_{12}(\vec{x}, \vec{y}, t)=0 \quad, \quad \mathcal{H}_{21}(\vec{x}, \vec{y}, t)=0
\end{gathered}
$$

The static HB solution is caracterized by $w_{0}=0$, i. e. in the uniform case

$$
\left(m_{0}^{2}+\frac{b}{6} \varphi_{0}+\frac{b}{2} G_{0}(x, x)\right) \varphi_{0}=0
$$

and the gap equation

$$
\Xi_{0}=-\tau \operatorname{coth}\left(\beta \mathcal{H}_{0} \tau\right)
$$

which can also be written as

$$
\beta \mathcal{H}_{0}=\frac{1}{2} \tau \ln \left(\frac{\Xi_{0}+\tau}{\Xi_{0}-\tau}\right)
$$

More explicitly, in the uniform case, by working in the momentum space,

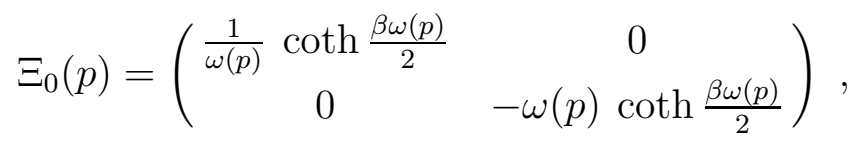

with $\omega^{2}(p)=p^{2}+m_{0}^{2}+\frac{b}{2} \varphi_{0}^{2}+\frac{b}{2} G_{0}(\vec{x}, \vec{x})$.

In the following we will show that the mean-field equations (2.10) and (2.11) have a Poisson structure.

\section{The Poisson tensor and the Poisson structure of the mean-field equations}

We consider first the uniform case. A more general construction of the Poisson tensor in the non-uniform case will be given in the next section. We gather in a single vector $z^{\mu}, \mu=1,2,3$ the expectation values $<1>, \alpha_{j}$ and $\Xi_{i j}(p)$, where $\Xi_{i j}(p)$ is the Fourrier transformed of $\Xi(\vec{x}, \vec{y}, t)$ :

$$
\Xi_{i j}(\vec{x}, \vec{y})=\int \frac{d^{d} p}{(2 \pi)^{d}} e^{i \vec{p} \cdot(\vec{y}-\vec{x})} \Xi_{i j}(p) .
$$


We define the Poisson tensor $\mathcal{C}^{\mu \nu} \equiv\left\{z^{\mu}, z^{\nu}\right\}$ in the following way :

$$
\begin{aligned}
& \mathcal{C}_{i j}^{22}=\tau_{i j} \quad, \quad \mathcal{C}_{i, j k}^{23}=0 \\
& \mathcal{C}_{11,12}^{33}(p)=\mathcal{C}_{11,21}^{33}(p)=4 \Xi_{11}(p) \\
& \mathcal{C}_{12,11}^{33}(p)=\mathcal{C}_{21,11}^{33}(p)=-4 \Xi_{11}(p) \\
& \mathcal{C}_{11,22}^{33}(p)=8 \Xi_{12}(p) \\
& \mathcal{C}_{22,11}^{33}(p)=-8 \Xi_{12}(p) \\
& \mathcal{C}_{12,22}^{33}(p)=\mathcal{C}_{21,22}^{33}(p)=4 \Xi_{22}(p) \\
& \mathcal{C}_{22,12}^{33}(p)=\mathcal{C}_{22,21}^{33}(p)=-4 \Xi_{22}(p) \\
& \mathcal{C}_{12,12}^{33}(p)=\mathcal{C}_{12,21}^{33}(p)=0
\end{aligned}
$$

The other matrix elements of this tensor are equal to zero. $\mathcal{C}^{22}$ is a constant in the momentum space, while $\mathcal{C}^{33}$ depends on the momentum $p$. This Poisson tensor is a linear function of the dynamical variables $\Xi_{i j}(p)$. Let us note that it does not depend on the value of the condensat $\alpha$.

The Poisson structure associated to the Poisson tensor $\mathcal{C}^{\mu \nu}$ is defined according to

$$
\{f, g\} \equiv \frac{1}{i} \frac{\partial f}{\partial z^{\mu}} \mathcal{C}^{\mu \nu} \frac{\partial g}{\partial z^{\nu}}
$$

for any functions $f$ and $g$ of the dynamical variables $z^{\mu}$.

We check that the operation $\{f, g\}=k$ which associates to two functions $f$ and $g$ a third function $k$ is bilinear and antisymmetric. It satisfies also Jacobi's identity and Leibnitz's derivation rule [9]. (When the Poisson structure is not defined from a Lie algebra, we have to check these properties.)

In particular, we have

$$
\left\{\alpha_{i}, g\right\}=\frac{1}{i} \mathcal{C}_{i j}^{22} \frac{\partial g}{\partial \alpha_{j}}=\frac{1}{i} \tau_{i j} \frac{\partial g}{\partial \alpha_{j}} .
$$

From the definition $w_{i} \equiv \frac{\partial \mathcal{E}}{\partial \alpha_{i}}$, we can write the first TDHB equation (2.10) as :

$$
\dot{\alpha}_{i}=\left\{\alpha_{i}, \mathcal{E}\right\}
$$

We have also :

$$
\left\{\Xi_{i j}, g\right\}=\frac{1}{i} \mathcal{C}_{i j, k l}^{33} \frac{\partial g}{\partial \Xi_{k l}}
$$

If we choose $g=\mathcal{E}$, with the definition (3.2) of $\mathcal{C}^{33}$ and the definition (2.14) of the matrix $\mathcal{H}$, we obtain :

$$
\begin{aligned}
& i\left\{\Xi_{11}, \mathcal{E}\right\}=-4 \Xi_{12}(p) \mathcal{H}_{22}(p) \\
& i\left\{\Xi_{12}, \mathcal{E}\right\}=2 \Xi_{11}(p) \mathcal{H}_{11}(p)-2 \Xi_{22}(p) \mathcal{H}_{22}(p) . \\
& i\left\{\Xi_{22}, \mathcal{E}\right\}=4 \Xi_{12}(p) \mathcal{H}_{11}(p)
\end{aligned}
$$


The second TDHB equation (2.11) can be put in the form :

$$
\dot{\Xi}_{i j}=\left\{\Xi_{i j}, \mathcal{E}\right\}
$$

The TDHB equations in the $\Phi^{4}$ theory can therefore be written as classical dynamical equations, the mean-field energy density $\mathcal{E}(\alpha, \Xi)$ playing the role of a classical Hamiltonian with the non-symplectic Poisson structure defined by (3.2).

It is actually more useful to consider the free-energy density

$$
\mathcal{F}(\alpha, \Xi)=\mathcal{E}(\alpha, \Xi)-\beta^{-1} \mathcal{S}(\Xi)
$$

as a classical Hamiltonian instead of $\mathcal{E}$. The von-Neuman entropy density $\mathcal{S}$ is defined by

$$
\mathcal{S} \equiv-\frac{\operatorname{Tr} \mathcal{D} \log \mathcal{D}}{\operatorname{Tr} \mathcal{D}}+\log \operatorname{Tr} \mathcal{D}
$$

For a gaussian density matrix it can be written as a function of the matrix $\Xi$ as :

$$
\begin{aligned}
\mathcal{S}=\int \frac{d^{3} p}{(2 \pi)^{3}} \quad & {\left[\frac{1}{4} \operatorname{tr}\left\{\left(\frac{1}{2}(1-\tau) \Xi(1-\tau)-1\right) \log \left(\frac{\Xi+\tau}{\Xi-\tau}\right)\right\}\right.} \\
& \left.+\frac{1}{2} \log \operatorname{det}\left\{\frac{1}{4}\left(\begin{array}{cc}
1 & 1 \\
1 & -1
\end{array}\right) \Xi(1-\tau)-\frac{1}{2}\left(\begin{array}{ll}
0 & 1 \\
1 & 0
\end{array}\right)\right\}\right],
\end{aligned}
$$

or

$$
\mathcal{S}=\int \frac{d^{3} p}{(2 \pi)^{3}}\left[\frac{1}{2} \sqrt{I} \log \left(\frac{\sqrt{I}+1}{\sqrt{I}-1}\right)+\frac{1}{2} \log (I-1)\right],
$$

where $\mathrm{I}$ is the Heisenberg invariant

$$
\begin{aligned}
& I(x, y)=\int d^{3} z \quad(4<\bar{\Phi}(x) \bar{\Phi}(z)><\bar{\Pi}(z) \bar{\Pi}(y)> \\
& -<\bar{\Phi}(x) \bar{\Pi}(z)+\bar{\Pi}(z) \bar{\Phi}(x)><\bar{\Phi}(z) \bar{\Pi}(y)+\bar{\Pi}(y) \bar{\Phi}(z)>) \quad \\
& I(x, y)=\int d^{3} z\left(-\Xi_{11}(x, z) \Xi_{22}(z, y)+\Xi_{12}(x, z) \Xi_{12}(z, y)\right) .
\end{aligned}
$$

For a pure state, we have : $\Xi_{11} \Xi_{22}-\Xi_{12}^{2}+1=0$ or $I(x, y)=\delta(x-y)$.

We have $\{\mathcal{S}(\Xi), \alpha\}=0$ and $\{\mathcal{S}(\Xi), \Xi\}=0$. Therefore, the TDHB equations (3.5) and (3.8) can be written as :

$$
\begin{aligned}
\dot{\alpha}_{i} & =\left\{\alpha_{i}, \mathcal{F}(\alpha, \Xi)\right\}, \\
\dot{\Xi}_{i j} & =\left\{\Xi_{i j}, \mathcal{F}(\alpha, \Xi)\right\}
\end{aligned}
$$

The free-energy density $\mathcal{F}(\alpha, \Xi)$ is a constant of the motion like $\mathcal{E}(\alpha, \Xi)$ and $\mathcal{S}(\Xi)$. The static solution $\alpha_{0}, \Xi_{0}$ (see eq. (2.20) and (2.21)) of the TDHB equations is a minimum of $\mathcal{F}$ (at zero temperature, it is a minimum of $\mathcal{E}$ ). In section 6 , we will derive the linearized mean-field equations arround the static solution. 


\section{Définition of the Poisson tensor from a Lie algebra}

In this section we consider the non uniforme case and we work with the space indices. We define the following operators $\mathcal{O}^{\mu}$ :

$$
\begin{gathered}
\mathcal{O}^{1}=1 \\
\mathcal{O}^{2}(\vec{x})=\left(\begin{array}{c}
\Phi(\vec{x}) \\
-i \Pi(\vec{x})
\end{array}\right) \\
\mathcal{O}^{3}(\vec{x}, \vec{y})=\left(\begin{array}{cc}
2 \bar{\Phi}(\vec{x}) \bar{\Phi}(\vec{y}) & -i(\bar{\Phi}(\vec{x}) \bar{\Pi}(\vec{y})+\bar{\Pi}(\vec{y}) \bar{\Phi}(\vec{x})) \\
-i(\bar{\Phi}(\vec{y}) \bar{\Pi}(\vec{x})+\bar{\Pi}(\vec{x}) \bar{\Phi}(\vec{y})) & -2 \bar{\Pi}(\vec{x}) \Pi(\vec{y})
\end{array}\right)
\end{gathered}
$$

where $\overline{\mathcal{O}}=\mathcal{O}-<\mathcal{O}>$ for any operator $\mathcal{O}$.

It is easy to show that these operators satisfy a Lie algebra :

$$
\left[\mathcal{O}^{\mu}, \mathcal{O}^{\nu}\right]=f_{\sigma}^{\mu \nu} \mathcal{O}^{\sigma}
$$

More explicitly :

$$
\begin{gathered}
{\left[\mathcal{O}_{i}^{2}(\vec{x}), \mathcal{O}_{j}^{2}(\vec{y})\right]=\left(f_{1}^{22}\right)_{i j}(\vec{x}, \vec{y}) \mathcal{O}^{1}} \\
{\left[\mathcal{O}_{i}^{2}(\vec{x}), \mathcal{O}_{j k}^{3}(\vec{y}, \vec{z})\right]=\int d^{d} u \sum_{m}\left(f_{2}^{23}\right)_{i, j k}^{m}(\vec{x}, \vec{y}, \vec{z} ; \vec{u}) \mathcal{O}_{m}^{2}(\vec{u})+\left(f_{1}^{23}\right)_{i, j k}(\vec{x}, \vec{y}, \vec{z}) \mathcal{O}^{1}} \\
{\left[\mathcal{O}_{i j}^{3}(\vec{x}, \vec{y}), \mathcal{O}_{k l}^{3}(\vec{z}, \vec{t})\right]=\int d^{d} u d^{d} v \sum_{m, n}\left(f_{3}^{33}\right)_{i j, k l}^{m n}(\vec{x}, \vec{y}, \vec{z}, \vec{t} ; \vec{u}, \vec{v}) \mathcal{O}_{m n}^{3}(\vec{u}, \vec{v})}
\end{gathered}
$$

The structure constants $f_{1}^{22}, f_{2}^{23}, f_{3}^{33}$ are delta functions in position space while $f_{1}^{23}$ depends also on the expectation values $\left\langle\mathcal{O}_{i}^{2}(\vec{x})\right\rangle$. The other structure constants $f_{\sigma}^{\mu \nu}$ are equal to zero. For instance, we have :

$$
f_{1}^{22}(\vec{x}, \vec{y})=\tau \delta(\vec{x}-\vec{y}) \quad, \quad\left(f_{1}^{23}\right)_{1,12}(\vec{x}, \vec{y}, \vec{z})=-2 \varphi(\vec{x}) \delta(\vec{x}-\vec{y}) \delta(\vec{x}-\vec{z}) .
$$

We then consider the expectation values $z^{\mu} \equiv<\mathcal{O}^{\mu}>$ and we define the Poisson bracket $\mathcal{C}^{\mu \nu} \equiv\left\{z^{\mu}, z^{\nu}\right\}$ from the Lie algebra characterized by the structure constant $f$ :

$$
\mathcal{C}^{\mu \nu} \equiv\left\{z^{\mu}, z^{\nu}\right\}=f_{\sigma}^{\mu \nu} z^{\sigma}
$$

Similarly to the structure constant $f$, the tensor $\mathcal{C}^{\mu \nu}$ has space indices.

In the uniform case, by working in the momentum space, we can show that the only non-vanishing commutators between the operators $\mathcal{O}^{\mu}$ can be written as :

$$
\begin{gathered}
{\left[\mathcal{O}_{i}^{2}, \mathcal{O}_{j}^{2}\right]=\tau_{i j}} \\
{\left[\mathcal{O}_{i}^{2}, \mathcal{O}_{j k}^{3}(\vec{p}=0)\right]=\sum_{m}\left(f_{2}^{23}\right)_{i, j k}^{m} \mathcal{O}_{m}^{2}+\left(f_{1}^{23}\right)_{i, j k} \mathcal{O}^{1}} \\
{\left[\mathcal{O}_{i j}^{3}(\vec{p}), \mathcal{O}_{k l}^{3}(\vec{p})\right]=\sum_{m n}\left(f_{3}^{33}\right)_{i j, k l}^{m n} \mathcal{O}_{m n}^{3}(\vec{p})}
\end{gathered}
$$

where the structure constants $f$ do not depend on the momentum $\vec{p}$. In this way we find again the Poisson tensor defined in section 3. 


\section{$5 \quad$ Heisenberg Invariant}

Another presentation of the Poisson tensor can be useful to find the structural invariants . We still consider the uniform case and we work in the momentum space. By chosing the coordinates $<\Phi>,<\Pi>,<\bar{\Phi} \bar{\Phi}>(p),<\bar{\Pi} \bar{\Pi}>(p),<\bar{\Phi} \bar{\Pi}+\bar{\Pi} \bar{\Phi}>(p)$, the Poisson tensor can be written as a $5 \times 5$ matrix, build from two blocks : the symplectic matrix $\left(\begin{array}{cc}0 & 1 \\ -1 & 0\end{array}\right)$ and the $3 \times 3$ antisymmetric matrix defined by the elements :

$$
\begin{aligned}
& \tilde{\mathcal{C}}_{11,12}^{33}(p) \equiv\{<\bar{\Phi} \bar{\Phi}>(p),<\bar{\Phi} \bar{\Pi}+\bar{\Pi} \bar{\Phi}>(p)\}=4<\bar{\Phi} \bar{\Phi}>(p) \\
& \tilde{\mathcal{C}}_{12,22}^{33}(p) \equiv\{<\bar{\Phi} \bar{\Pi}+\bar{\Pi} \bar{\Phi}>(p),<\bar{\Pi} \bar{\Pi}>(p)\}=4<\bar{\Pi} \bar{\Pi}>(p) \\
& \tilde{\mathcal{C}}_{11,22}^{33}(p) \equiv\{<\bar{\Phi} \bar{\Phi}>(p),<\bar{\Pi} \bar{\Pi}>(p)\}=2<\bar{\Phi} \bar{\Pi}+\bar{\Pi} \bar{\Phi}>(p)
\end{aligned}
$$

that is

$$
M=\left(\begin{array}{ccc}
0 & 2<\bar{\Phi} \bar{\Pi}+\bar{\Pi} \bar{\Phi}>(p) & 4<\bar{\Phi} \bar{\Phi}>(p) \\
-2<\bar{\Phi} \bar{\Pi}+\bar{\Pi} \bar{\Phi}>(p) & 0 & -4<\bar{\Pi} \bar{\Pi}>(p) \\
-4<\bar{\Phi} \bar{\Phi}>(p) & 4<\bar{\Pi} \bar{\Pi}>(p) & 0
\end{array}\right)
$$

The rank of the Poisson tensor is a function of the dynamical variables. Near a regular point, the rank of the Poisson tensor is constant. We can find coordinates $(\mathrm{Q}, \mathrm{P}, \mathrm{C})$ such as the matrix $M$ can put in the form :

$$
M^{\prime}=\left(\begin{array}{ccc}
0 & 1 & 0 \\
-1 & 0 & 0 \\
0 & 0 & 0
\end{array}\right)
$$

The Poisson brackets in this coordinates system are given by the symplectic form

$$
\{f, g\}=\frac{\partial f}{\partial Q} \frac{\partial g}{\partial P}-\frac{\partial f}{\partial P} \frac{\partial g}{\partial Q}
$$

We have therefore locally a canonical Hamiltonian system which depends on one parameter $\mathrm{C}$. The function of the dynamical variables which is equal to $\mathrm{C}$ is a Casimir function: it satisfies $\{f, C\}=0$ for any function $f$ of the dynamical variables [2]

The coordinate $\mathrm{C}$ corresponding to the eigenvalue 0 of the Poisson tensor defined by the matrix (5.4) is :

$$
C(p)=4<\bar{\Phi} \bar{\Phi}>(p)<\bar{\Pi} \bar{\Pi}>(p)-<\bar{\Phi} \bar{\Pi}+\bar{\Pi} \bar{\Phi}>(p)<\bar{\Phi} \bar{\Pi}+\bar{\Pi} \bar{\Phi}>(p) .
$$

We recognize the Heisenberg invariant (3.13) and we have :

$$
\dot{C}(p)=0
$$




\section{Linearized equations}

At the HB solution $z_{0}^{\mu}$ which is a regular point of the Poisson tensor, the matrix defined by :

$$
\left.\mathcal{B}^{\mu \nu} \equiv \frac{\partial^{2} \mathcal{F}}{\partial z^{\mu} \partial z^{\nu}}\right|_{z_{0}}
$$

is positive definite. The free-energy density $\mathcal{F}$ being conserved by the flow defined with the Poisson brackets (3.3), this implies that $z_{0}^{\mu}$ is a stable equilibrium point.

The TDHB equations write :

$$
\begin{gathered}
\dot{\alpha}_{i}=\left\{\alpha_{i}, \mathcal{F}\right\}=\frac{1}{i} \mathcal{C}_{i j}^{22}(\Xi) \frac{\partial \mathcal{F}}{\partial \alpha_{j}} \\
\dot{\Xi}_{i j}=\left\{\Xi_{i j}, \mathcal{F}\right\}=\frac{1}{i} \mathcal{C}_{i j, k l}^{33}(\Xi) \frac{\partial \mathcal{F}}{\partial \Xi_{k l}}
\end{gathered}
$$

where we have explicitly written the dependence of the Poisson tensor on the matrix $\Xi$. Let us develop these equations arround the static HB solution $\alpha_{0}, \Xi_{0}$ at the first order in $\delta \alpha=\alpha-\alpha_{0}$ and $\delta \Xi=\Xi-\Xi_{0}$. Because $\alpha_{0}, \Xi_{0}$ is a minimum of $\mathcal{F}$, the variation of $\mathcal{C}$ arround $\alpha_{0}, \Xi_{0}$ does not contribute. By introducing

$$
\mathcal{F}^{(2)}(\alpha, \Xi)=\mathcal{F}^{(2)}\left(\alpha_{0}, \Xi_{0}\right)+\left.\frac{1}{2} \delta z^{\mu} \delta z^{\nu} \frac{\partial^{2} \mathcal{F}}{\partial z^{\mu} \partial z^{\nu}}\right|_{z_{0}}
$$

we obtain :

$$
\begin{gathered}
\frac{d \delta \alpha_{i}}{d t}=\left\{\delta \alpha_{i}, \mathcal{F}^{(2)}(\alpha, \Xi)\right\}_{0}=\frac{1}{i} \mathcal{C}_{i j}^{22}\left(\Xi_{0}\right) \frac{\partial \mathcal{F}^{(2)}}{\partial\left(\delta \alpha_{j}\right)} \\
\frac{d \delta \Xi_{i j}}{d t}(q)=\left\{\delta \Xi_{i j}, \mathcal{F}^{(2)}(\alpha, \Xi)\right\}_{0}=\frac{1}{i} \mathcal{C}_{i j, k l}^{33}\left(\Xi_{0}\right)(q) \frac{\partial \mathcal{F}^{(2)}}{\partial\left(\delta \Xi_{k l}\right)}
\end{gathered}
$$

where the indice 0 for the Poisson bracket indicates that the Poisson tensor $\mathcal{C}$ has to be evaluated at the point $\Xi_{0}$. In this way, we obtain the small oscillations equations arround the static HB solution also called RPA equations. These equations are of Hamilton type with the free energy developped to second order $\mathcal{F}^{(2)}(\alpha, \Xi)$ playing the role of the hamiltonian and with $\mathcal{C}\left(\Xi_{0}\right)$ as Poisson tensor. $\mathcal{F}^{(2)}$ is a constant of the linearized equations of motion and has a local minimum at $\alpha_{0}, \Xi_{0}$. Therefore the linearized dynamical equations at $z_{0}^{\mu}$ have 0 as stable equilibrium.

The small oscillations equations (6.5) and (6.6) can be written as :

$$
\begin{gathered}
i \frac{d \delta \alpha_{i}}{d t}=\mathcal{C}_{i j}^{22}\left(\Xi_{0}\right)\left(\mathcal{B}_{j k}^{22} \delta \alpha_{k}+\int \frac{d^{3} q}{(2 \pi)^{3}} \mathcal{B}_{j, k l}^{23}(q) \delta \Xi_{l k}(q)\right) \\
i \frac{d \delta \Xi_{i j}(q)}{d t}=\mathcal{C}_{i j, k l}^{33}\left(\Xi_{0}\right)(q)\left(\mathcal{B}_{l k, m}^{32}(q) \delta \alpha_{m}+\int \frac{d^{3} k}{(2 \pi)^{3}} \mathcal{B}_{l k, m n}^{33}(q, k) \delta \Xi_{n m}(k)\right)
\end{gathered}
$$


The expression of the matrix elements of $\mathcal{B}^{\mu \nu}$ in the $\Phi^{4}$ theory is given in appendix. In equations (6.7) and (6.8), we have considered small oscillations arround the uniform static HB solution :

$$
\begin{gathered}
\alpha(\vec{p}, t)=\alpha_{0} \delta(\vec{p})+\delta \alpha(\vec{p}, t) \\
\Xi\left(\vec{p}_{1}, \vec{p}_{2}, t\right)=\Xi_{0}\left(\vec{p}_{1}\right) \delta\left(\vec{p}_{1}+\vec{p}_{2}\right)+\delta \Xi\left(\vec{p}_{1}, \vec{p}_{2}, t\right) .
\end{gathered}
$$

where

$$
\Xi\left(\vec{p}_{1}, \vec{p}_{2}, t\right)=\int d^{3} x d^{3} y e^{-i \overrightarrow{p_{1}} \cdot \vec{x}-i \overrightarrow{p_{2}} \cdot \vec{y}} \Xi(\vec{x}, \vec{y}, t) .
$$

We have then introduced the total momentum $\vec{P}=\vec{p}_{1}+\vec{p}_{2}$ and the relative momentum $\vec{q}=\frac{1}{2}\left(\vec{p}_{1}-\vec{p}_{2}\right)$ and we have written the equations for a total momentum $\vec{P}=\overrightarrow{0}$.

Equations (6.5) and (6.6) could also have been obtained by linearizing the original expressions (2.10) and (2.11) arround the static solution :

$$
\begin{gathered}
i \delta \dot{\alpha}=\tau \delta w \\
i \delta \dot{\Xi}=2(\delta \Xi \mathcal{H} \tau-\tau \mathcal{H} \delta \Xi)+2\left(\Xi_{0} \delta \mathcal{H} \tau-\tau \delta \mathcal{H} \Xi_{0}\right) .
\end{gathered}
$$

We define the matrices $t, T, r, R$ (which are the analogs of the RPA matrix $\mathcal{R}$ of reference [8]) by expanding $<H>$ up to the second order, or the vector $w$ and the matrix $\mathcal{H}$ up to the first order :

$$
\begin{aligned}
\delta w_{i}(\vec{x}, t) & =\int d^{d} y t_{i j}(\vec{x}, \vec{y}, t) \delta \alpha_{j}(\vec{y}, t)-\frac{1}{2} \int d^{d} y d^{d} z T_{i, j k}(\vec{x}, \vec{y}, \vec{z}, t) \delta \Xi(\vec{z}, \vec{y}, t) \\
\delta \mathcal{H}_{i j}(\vec{x}, \vec{y}, t) & =\int d^{d} z r_{i j, k}(\vec{x}, \vec{y}, \vec{z}, t) \delta \alpha_{k}(\vec{z}, t)-\frac{1}{2} \int d^{d} z d^{d} u R_{i j, k l}(\vec{x}, \vec{y}, \vec{z}, \vec{u}, t) \delta \Xi_{l k}(\vec{u}, \vec{z}, t) .
\end{aligned}
$$

We therefore obtain a relation between these matrices and the matrix $\mathcal{B}$ of the second derivatives of the free energy at the minimum. This relation involves the Poisson tensor evaluated at $\Xi_{0}$.

From the equations (6.7) and (6.8), we can study the existence of bound states at finite temperature both in the symmetric phase and in the broken phase. This will be a generalization to finite temperature of the results obtained by Kerman and Lin at zero temperature [10].

To conclude, we have shown that the time-dependent mean-field equations in $\Phi^{4}$ theory can be written as classical dynamical equations with a non-symplectic Poisson structure, the free-energy density playing the role of a classical Hamiltonian. The Heisenberg invariant appears as a structural invariant of the Poisson structure. This Poisson structure can be useful to discuss stability properties of trajectories characterized by the condensat and the fluctuations of the quantum field at finite temperature. The occurrence of a vanishing eigenvalue for the matrix $\mathcal{B}$ at a critical temperature for a given coupling 
constant is the signal of a phase transition from the phase with broken symmetry to the symmetric phase.

\section{Aknowledgements}

I wish to thank Marcel Vénéroni for very helpful conversations.

\section{Appendix}

In this appendix we give the expression of the elements of the matrix $\mathcal{B}$ in the case of the $\Phi^{4}$ theory.

$$
\begin{gathered}
\mathcal{B}_{11}^{22}=m_{0}^{2}+\frac{b}{2} \varphi_{0}^{2}+\frac{b}{2} G_{0}(\vec{x}, \vec{x}) \quad, \quad \mathcal{B}_{22}^{22}=-1 \quad, \quad \mathcal{B}_{12}^{22}=\mathcal{B}_{21}^{22}=0 \\
\mathcal{B}_{1,11}^{23}=\frac{\partial^{2} \mathcal{E}}{\partial \alpha_{1} \partial \Xi_{11}(p)}=\frac{b}{4} \varphi_{0} \\
\mathcal{B}_{i j, k l}^{33}(p, q)=\frac{\partial^{2} \mathcal{E}}{\partial \Xi_{j i}(p) \partial \Xi_{l k}(q)}-\frac{1}{\beta} \frac{\partial^{2} \mathcal{S}}{\partial \Xi_{j i}(p) \partial \Xi_{l k}(q)} \delta^{3}(p-q) \\
\mathcal{B}_{11,11}^{33}(p, q)=\frac{b}{16}+\frac{1}{4 \beta} \omega^{2}(p)\left(\frac{x}{\operatorname{coth} x}+\frac{1}{\operatorname{coth}^{2} x-1}\right) \delta^{3}(p-q) \\
\mathcal{B}_{11,22}^{33}(p, q)=\frac{1}{4 \beta}\left(\frac{x}{\operatorname{coth} x}-\frac{1}{\operatorname{coth}{ }^{2} x-1}\right) \delta^{3}(p-q) \\
\mathcal{B}_{22,22}^{33}(p, q)=\frac{1}{4 \beta \omega^{2}(p)}\left(\frac{x}{\operatorname{coth} x}+\frac{1}{\operatorname{coth}^{2} x-1}\right) \delta^{3}(p-q) \\
\mathcal{B}_{12,12}^{33}(p, q)=-\frac{1}{\beta} \frac{x}{\operatorname{coth} x} \delta^{3}(p-q)
\end{gathered}
$$

where $x=\frac{\beta \omega(p)}{2}$ and

$$
\omega^{2}(p)=p^{2}+m_{0}^{2}+\frac{b}{2} \varphi_{0}^{2}+\frac{b}{2} G_{0}(\vec{x}, \vec{x}, t)
$$

The other elements of the matrix $\mathcal{B}$ are equal to zero.

\section{References}

[1] O. Eboli, R. Jackiw and S. Y. Pi, Phys. Rev. D37 (1988) 3557

[2] A. Weinstein, p. 3 in "Fluids and Plasmas : Geometry and dynamics", J. E. Marsden (Ed) Contemporary Mathematics, Vol.28, Amer. Math. Soc., Providence, RI,1984.

[3] I. E. Dzyaloshinskii and G. E. Volovick, Ann. of Phys. 125 (1980) 67 
[4] J. E. Marsden, R. Montgomery, P. J. Morrison and W. B. Thompson, Ann. of Phys. 169 (1986) 29

[5] R. Balian and M. Vénéroni, Ann. of Phys. 195 (1989) 324

[6] M. Benarous, Ann. of Phys. 264 (1998) 1

[7] C. Martin, Phys. Rev. D52 (1995) 7121

[8] R. Balian and M. Vénéroni, Ann. of Phys. 187 (1988) 29

[9] P. A. M. Dirac, "Lectures on Quantum Mechanics", Belfer Graduate School of Science Monographs, Yeshiva University, New York, 1964.

[10] A. K.Kerman and C. Y. Lin, Ann. of Phys. 241 (1995) 185; hep-th/9805061 